\title{
Technical considerations concerning digital technologies
}

Luís Lança and Augusto Silva

\begin{abstract}
This chapter addresses technical issues concerning digital technologies. Radiological equipment and technique are briefly introduced together with a discussion about requirements and advantages of digital technologies. Digital technologies offer several advantages when compared to conventional analogical systems, or screen-film (SF) systems. While in clinical practice the practitioners should be aware of technical factors such as image acquisition, management of patient dose, and diagnostic image quality. Thus, digital technologies require an up-to-date scientific knowledge concerning their use in projection radiography. In this chapter, technical considerations concerning digital technologies are provided.
\end{abstract}

Keywords; Technique; Digital technology; Radiological equipment; Requirements; Advantages; Conventional; Analog; Screen-film systems; Clinical practice; Image acquisition; Projection radiography. 